\title{
EXPERIMENTAL RESEARCH AND JUSTIFICATION OF PARAMETERS OF SPIRAL POTATO CLEANER FROM ADMIXTURES
}

\author{
Volodymyr Bulgakov ${ }^{1}$, Zinoviy Ruzhylo ${ }^{1}$, Ivan Fedosiy ${ }^{1}$, Semjons Ivanovs ${ }^{2}$ \\ ${ }^{1}$ National University of Life and Environmental Sciences of Ukraine, Ukraine; ${ }^{2}$ Latvia University of \\ Life Sciences and Technologies, Latvia \\ semjons@apollo.lv
}

\begin{abstract}
When digging potato tubers from the soil, one of the main problems is separation of a large amount of the soil and plant admixtures from the heap, which is fed into the potato harvester. We have developed a new design for a potato heap cleaner that consists of five cantilever-fixed cleaning driving spirals, arranged in a wave-like manner, significantly expanding the working zone of separation of the incoming potato heaps, and this, in turn, will contribute to its better dispersal over the working surface of the cleaner, more intense destruction of the soil lumps, and, consequently, improved screening of the soil and plant admixtures, and reduced clogging of the cleaning spirals. The experimental studies conducted under the field conditions showed that the process of cleaning tubers from the soil and plant admixtures, using this cleaner, occurs due to intense movement of the potato heaps by means of the coils of the cantilever-fixed cleaning spiral springs. The springs are rotating at a pre-set angular velocity, making oscillatory movements because of the deflection of their longitudinal axes under the impact of the weight of the heap arriving into the working zone of the cleaner. There was built a mathematical model of the movement of the body of a potato across the surface of the spiral separator. In addition, there was analytically considered the relative motion of a single body of the potato tuber across the surface of the spiral coil with a pre-set radius and angle of the pitch of the helix. As a result of mathematical simulation, a system of differential equations of the movement was compiled, the solution of which made it possible to determine, at the pre-set design parameters, the optimal kinematic characteristics that ensure the guaranteed movement of the potato tuber across the surface of the cleaning spiral spring.
\end{abstract}

Keywords: potato, cleaner, admixtures, parameters.

\section{Introduction}

Potato growing is an important agricultural sector in many countries, including Ukraine, Belarus and the Baltic countries [1-3].

Potato harvesting is the most time-consuming operation in the technological cycle. One of the important problems when harvesting potatoes is cleaning of its tubers from the soil admixtures and plant residues. The solution to this problem will ensure not only the quality of the obtained products, but also eliminate the removal from the fields the soil which is fertile. Therefore, the development of potato cleaners from admixtures immediately after extracting the tuberous layer from the soil (potato tubers, soil admixtures, as well as rigid soil formations, rhizomes, left over tops, stones, etc.) is an important urgent scientific and technical problem in the field of agricultural engineering.

Many researchers have dealt with the problem of creating and optimising the potato tuber cleaners [4-7]. However, the problem how to improve the machines is still relevant. We have developed a new design of a potato heap cleaner [3], which consists of three cantilever driving spirals, which ensures an active cleaning surface of a wave-like shape, to which the dug-out heap is delivered. In addition, there is a possibility to change the angle of inclination of the indicated cleaning surface, which ensures the intensity of separation of admixtures and guarantees the movement of the tubers in the direction of the unloading elevator.

All this also contributes to better distribution of the heap over the working surface of the cleaner, more intensive destruction of the soil lumps, and, consequently, to better sifting down of soil and the plant admixtures and reducing clogging of the cleaning spirals. As a result, the cleaning efficiency and productivity of the potato cleaner from the soil and plant admixtures [8] increases. In order to optimise the parameters of this cleaner, it is necessary to conduct a series of investigations.

The purpose of this research is to substantiate the optimal design and kinematic parameters of a new spiral-type potato heap cleaner, using the results of an experimental multifactor field study.

\section{Materials and methods}

Structurally, the spiral cleaner of potatoes from admixtures consists of several driven cleaning spiral rollers 1 , made in the form of cantilever mounted spiral springs, and forming a wave-shaped 
cleaning surface. From one side a loading elevator 2 is connected to this cleaning surface, and an unloading elevator 3 is located on the opposite side. In addition, the upper part of the indicated cleaning surface has a fixed (on the frame) flat protective screen 4 , and, on the side of the unloading elevator 3 , there is fixed a flat protective screen 5 , which prevents the loss of the potato tubers. The rollers 1 consist of cantilever cleaning spirals having free ends 6 . The spirals themselves are mounted on the hubs 7 , which, in their turn, are connected to the drive shafts 8 .

a)

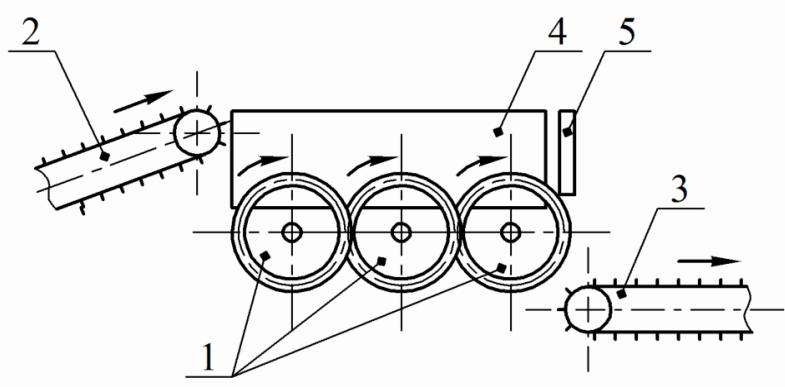

b)

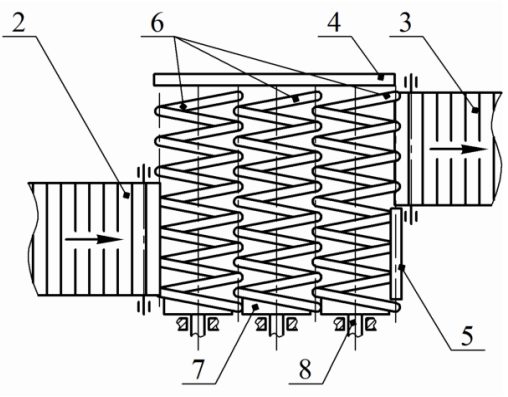

Fig. 1. Design of the spiral type potato cleaner: $a$ - side view; $b$ - top view;

1 - roller, consisting of the driving cleaning spirals; 2 - loading elevator; 3 - unloading elevator;

4 and 5 - protective screens; 6 - cantilever ends of the cleaning spirals;

7 - fixed ends of the spirals; 8 - shafts that rotate the spirals

The cleaning spirals of the rollers 1 mutually overlap. The entire cleaning surface, formed by the cleaning spirals of the rollers 1 , has a possibility to change its position, i.e. to change the angle of inclination to the horizon. The rotation of the three spiral cleaning spirals of the rollers 1 , ensured by the driving shafts 8 , may be set with various angular speeds, as a result of which the windings of the spirals may have different peripheral speeds. The direction of the rotational movement of the cleaning spirals of the rollers 1 is carried out in one direction. The cleaning spirals of the rollers 1 are installed with mutual overlap, but their free ends 6 can oscillate under the action of the variable load of the heap of potatoes and admixtures dug from the soil supplied by the loading elevator 2 .

Such a structural solution of this potato heap cleaner from the admixtures ensures significant gaps in its cleaning surface, which are formed by the spaces between the windings of the spirals themselves and the gaps between the windings of adjacent cleaning spirals. This increases the area, through which the separation of admixtures outside the cleaner takes place.

In addition, the advantages of this design include the absence of shafts in the middle of each cleaning spiral, which ensures unhindered downward passage of all the admixtures, and also prevents unwanted twisting of the plant residues. The hollowness of the internal spaces inside each spiral spring allows to increase the ability of forcible transportation of the entire mass of soil and plant residues to the outlet (cantilever) end of each spiral and drop it through the free end onto the field surface.

To prevent sticking of the soil to the cleaning spirals and the gaps between the spiral windings (moist soil), the spiral springs are installed with mutual overlap, in which the windings of one spiral partially enter the gaps of the other spiral. To ensure guaranteed movement of the potato tubers along the cleaning surface, which has a wavy shape, there is a possibility to change the angle of its inclination. Each cleaning spiral has an eccentricity, which allows it to make forced oscillatory movements in the process of work, which contribute to mixing of the heap fed onto it.

In order to implement this research, there were methods applied to conduct experimental field research, processing of the obtained data, the correlation and regression analyses, as well as methods for constructing nomograms [9-11].

\section{Results and discussion}

The previous studies $[3 ; 12]$ on the impact of the basic structural and kinematic parameters of a potato cleaner of the spiral-type upon the percentage of the sifted soil showed that, among the parameters of the cleaner that may affect separation of the soil and the admixtures, the greatest interest is in the study of the inclination angle $\alpha$ of the cleaner to the horizon (its spirals), the peripheral speed $V$ of the rotational movement of the spirals, the fixing eccentricity $\varepsilon$ of the spirals and the supply to the 
cleaning spirals of the material $Q$, i.e. heap of potato tubers with admixtures dug from the soil. Such design parameters of the cleaner as the diameter of the spiral $\left(D_{s}=133 \mathrm{~mm}\right)$, the angle of elevation of the helix of the spiral $\left(\gamma_{s}=12 \mathrm{deg}\right)$, the diameter of the rods of the spirals $\left(d_{s}=11 \mathrm{~mm}\right)$ were determined theoretically in our previous investigations [4] and, in this case, they were taken as constant values.

In order to conduct this research, a fractional factor experiment of the type $2^{4+1}$ was implemented with the repetition of each experiment 3 times. The model that was used to describe the impact of factors upon the optimisation parameter was of a linear kind. The regression equation of the technological process considered, expressed in the terms of coefficients in a general form, has the form:

$$
y=b_{0}+b_{1} \alpha+b_{2} V+b_{3} \varepsilon+b_{4} Q,
$$

where $b_{0}-b_{4}$ - regression coefficients.

After conducting the experimental field investigations and obtaining the data from these experiments, they were processed on a PC, using the Microsoft Excel 2003 application program. The results of processing the experimental data are presented in the form of a regression equation, which is a mathematical model of this process, linking the parameters of the cleaning working body with the indicators of its performance. We also compiled regression equations for all indicators of this technological process of cleaning the potato tubers from admixtures. In this case, the main parameter, when considering the indicated process, is admixtures sifted through the spiral cleaner.

Therefore, for this main indicator, i.e. percentage of the sifted soil, designated by us as $Y 1$, a regression equation was compiled, which has the following form:

$$
Y 1=118.396+0.25125 \alpha-12.2768 V+0.5325 \varepsilon-0.3175 Q,
$$

where $\alpha$-cleaner inclination angle to the horizon, deg;

$V$ - peripheral speed of rotational movement of the spirals, $\mathrm{m} \cdot \mathrm{s}^{-1}$;

$\varepsilon-$ eccentricity of spirals, $\mathrm{mm}$;

$Q$ - supply of material, $\mathrm{kg} \cdot \mathrm{s}^{-1}$.

The accuracy of expression (2) was reflected by the multiple correlation coefficient equal to $R=0.6883634$, and the determination coefficient $R$-squared $=0.780809$, the standard error of 4.556817 and the number of experiments equal to 8 . It should be noted that for such values of the correlation coefficient $R$ and determination coefficient $R^{2}$ can be judged on a fairly high accuracy of the obtained regression model. It was established that the correlation coefficient for the angle of inclination of the potato cleaner $\alpha$ to the horizon is: 0.421541 , for the peripheral speed $V$ of the spirals it is: -0.57674 , for the eccentricity $\varepsilon$ of fastening of the spirals, it is: 0.4467 , for the supply $Q$ it is: -0.26635 .

From the above results of experimental data processing a conclusion can be drawn that the peripheral rotation speed $V$ of the spirals has the greatest impact upon the percentage of sifted soil $Y 1$, and the eccentricity $\varepsilon$ and the angle of inclination $\alpha$ of the potato cleaner to the horizon have a slightly smaller effect. The smallest impact (with a weak connection) is made by an indicator - the supply of material $Q$. Here the minus sign in front of the coefficient indicates that with the growth of this factor increasing, the optimisation parameter decreases. Such an effect is produced by the angle of inclination $\alpha$ of the potato cleaner to the horizon and the supply of material $Q$

By its physical nature the sifting efficiency of the soil admixtures through the gaps between the turns of the spirals is determined exactly by the peripheral velocity of their rotational $V$ movement. And this will precisely provide the greatest impact upon the percentage of the sifted soil in comparison with the other variable coefficients. The intensive capture of the clods of soil by the turns of the spiral and their rapid movement into the gaps between the two adjacent spirals, and further sifting precisely ensure the intensification of this process.

Therefore, in the further laboratory experimental research the impact of the peripheral speed $V$ of the spirals and the angle of inclination $\alpha$ of the potato cleaner upon the percentage of the sifted soil and the separation intensity $Y 1$ at constant values of the eccentricity $\varepsilon$ of the spiral fastening and the supply of material $Q$ to the potato cleaner were studied. For this, a full-factor experiment $3^{2}$ was carried out 
with additional 4 points and fixed mass supply of $20 \mathrm{~kg} \cdot \mathrm{s}^{-1}$, fastening spirals with an eccentricity of $7 \mathrm{~mm}$. Statistical processing of the obtained results of the experimental studies was performed, using the Microsoft Excel 2003 application program.

Based on the obtained experimental data, a multivariate regression analysis was carried out, using different types of functions. The choice of the necessary function, by which a multivariate correlation analysis and an adequately mathematical model could be selected, was made by comparing the coefficients $D$ of multiple determination. Preference was given to the function with the highest value of this coefficient, which was a multidimensional polynomial of the second degree. For this case (i.e. for the case of a multidimensional polynomial of the second degree), we performed a regression analysis for the selected type of function (Table 1) with the number of factors 2 , variables 6 , with a probability level of $P=0.95$ (a fairly high degree of reproducibility) and $t_{\alpha}=2.176$ (the critical Student's coefficient) - for a polynomial of degree 2 .

After the exclusion of the insignificant factors, the regression equation has the following form:

$$
Y 1=66.9523+34.7557 \cdot V-0.0227 \cdot \alpha^{2}+0.3868 \cdot \alpha \cdot V-11.566691 \cdot V^{2} \text {, }
$$

at

$$
D=0.989, R=0.994, s=0.465 .
$$

Based on the obtained regression equation, using MathCad application program, we constructed a response surface of the percentage of the sifted soil $Y 1$ depending on the angle of inclination $\alpha$ of the potato cleaner and the peripheral speed of rotation $V$ of its spirals (Fig. 1).

Table 1

Results of the regression analysis

\begin{tabular}{|c|c|c|c|c|c|c|}
\hline Variable & Correlation & $\begin{array}{c}\text { Regression } \\
\text { coefficient } \\
\text { linearised }\end{array}$ & $\begin{array}{c}\text { Statistical } \\
\text { error of the } \\
\text { egression } \\
\text { coefficient }\end{array}$ & $\boldsymbol{t}_{\boldsymbol{\alpha}}$ & $\begin{array}{c}\text { Coefficient } \\
\text { of } \\
\text { elasticity }\end{array}$ & $\begin{array}{c}\text { Significance } \\
\text { of the } \\
\text { regression } \\
\text { coefficient }\end{array}$ \\
\hline \multicolumn{7}{|c|}{ Dependent variable } \\
\hline$Y 1$ & +67.2785 & & \\
\hline \multicolumn{7}{|c|}{ Independent variables } \\
\hline$V$ & +0.719 & -0.1397 & +0.18927 & -0.74 & -0.01 & insignificant \\
\hline$\alpha^{2}$ & -0.555 & +35.0949 & +15.72361 & +2.23 & +0.79 & significant \\
\hline$\alpha \cdot V$ & +0.578 & -0.0220 & +0.00293 & -7.51 & -0.04 & significant \\
\hline$V^{2}$ & -0.657 & +0.4466 & +0.08564 & +5.21 & +0.10 & significant \\
\hline
\end{tabular}

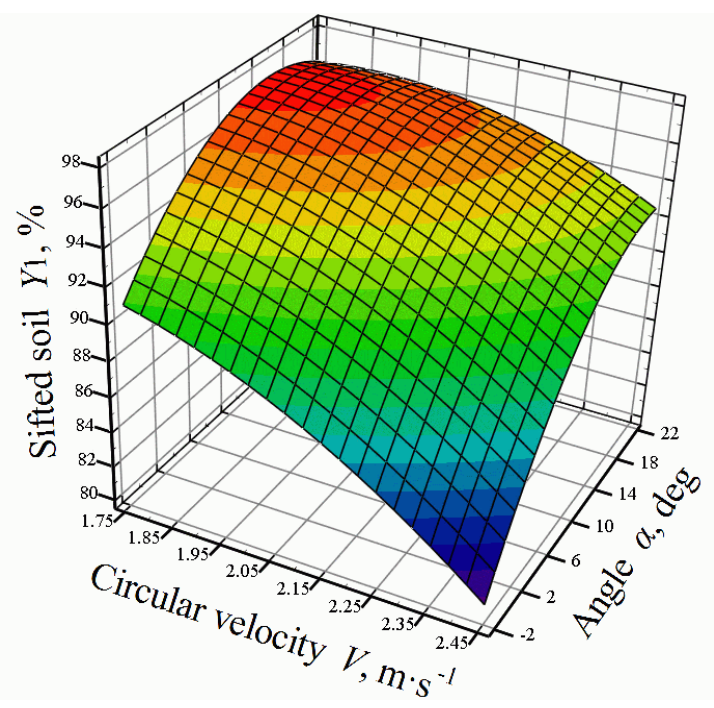

Fig. 1. Surface of the response of the impact of the angle of inclination $\alpha$ of the potato cleaner to the horizon and the peripheral speed $V$ of the rotational movement of the spiral upon the percentage of the sifted soil $Y 1$ 
We studied the extremum region in detail by the method of two-dimensional cross sections. For this, the regression equation was differentiated according to the angle of inclination $\alpha$ of the potato cleaner and the peripheral speed $V$ of its spirals.

The resulting partial derivatives are equated to zero, and a system of linear equations of the following form is obtained:

$$
\left.\begin{array}{l}
\frac{d \mathrm{Y} 1}{d \alpha}=-0.0454 \alpha+0.3868 V=0 \\
\frac{d \mathrm{Y} 1}{d V}=34.7557+0.3868 \alpha-23.13338 V=0 .
\end{array}\right\}
$$

Having solved the obtained system of equations (4), coordinates of the centre of the surface were obtained $\alpha \approx 14.82$ degrees, $V \approx 1.74 \mathrm{~m} \cdot \mathrm{s}^{-1}$. The optimisation criterion at this point is $Y 1 \approx 97.4 \%$.

Further, the regression equation was reduced to a canonical form. For this a characteristic equation is written, which has the following form:

$$
K^{2}+11.5894 K+0.1659=0 .
$$

As a result of solving this equation, it was found that: $K_{\alpha}=-0.0144, K_{V}=-11.5751$.

Since $\left|K_{\alpha}\right|<\left|K_{V}\right|$, then the contour curves (ellipses) that correspond to two-dimensional sections of the response surface are stretched out along the $\alpha$ axis, the axes are turned by the angle $\beta=0.96$ $\mathrm{rad}=55 \mathrm{deg}$ relative to the $V$ axis and, since the solutions of the characteristic equation are negative, the centre of the ellipses is the maximum point (Fig. 2).

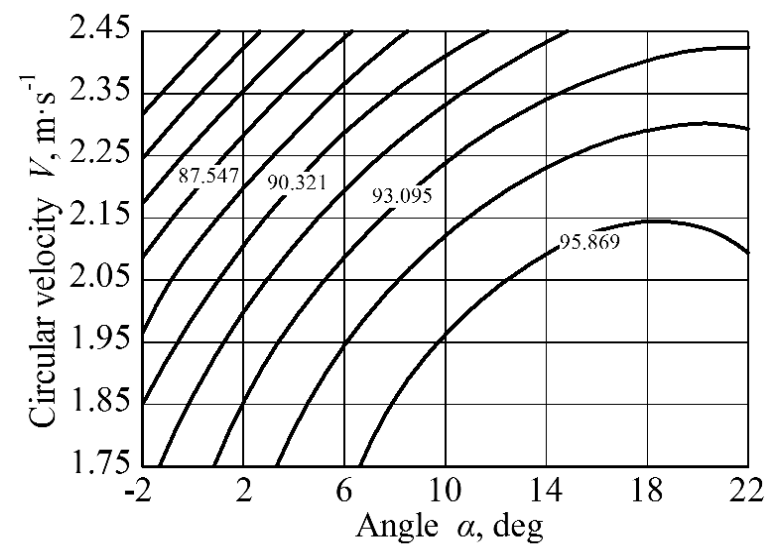

Fig. 2. Two-dimensional cross-section of the response surface of the impact of the angle of inclination $\alpha$ of the potato cleaner to the horizon and the peripheral speed $V$ of the rotational movement of the spiral upon the percentage of the sifted soil $Y 1$

Thus, in the laboratory experimental investigations the impact of such parameters of the spiral separator as the angle of inclination $\alpha$ to the horizon, the peripheral speed $V$ of the spirals, the eccentricity $\varepsilon$ of the fastening of the spirals and the supply of the material $Q$ to the percentage of sifted soil $Y 1$ was studied. The obtained research results allowed us to obtain regression equations according to the statistical data that are adequately described, and all the processes are reproducible. The rather high values of the multiple correlation coefficient indicate that the mathematical dependences (the type of functions) selected by us are consistent with the nature of the experimental dependencies. Consequently, a conclusion may be drawn that the obtained regression equations can be used to accurately determine the optimisation parameter and the optimal values of the factors studied.

Based on the obtained regression equations, a nomogram is constructed to determine the optimal parameters of the spiral separator. For this purpose, a nomogram is selected from aligned points with three rectilinear scales [12-14]. After certain transformations of the regression equations and taking the dependence on the angle of inclination $\alpha$ and the peripheral speed $V$ of the spirals through the parameter $P$ (for this parameter a dependence graph is plotted on the peripheral speed $V$ of the spirals for various values of the angle of inclination $\alpha$ of the potato cleaner), then, in our case, the function by which we can build a nomogram will have this form: 


$$
0.5325 \varepsilon-0.3175 Q=P .
$$

The variables that are in expression (6) vary within the following ranges: $\varepsilon=0-20 \mathrm{~mm}$, $Q=0-100 \mathrm{~kg} \cdot \mathrm{s}^{-1}, P=0-20$.

We accept that the height of the nomogram is $H=100 \mathrm{~mm}$, the width $L=80 \mathrm{~mm}$. For this type of nomograms the canonical appearance in the form of Cauchy will be the following:

$$
f_{1}+f_{2}=f_{3} .
$$

For our case $f_{1}=0.5325 \varepsilon, f_{2}=0.3175 Q$, and $f_{3}=P$.

The equation of the scales of the nomogram will have the following form:

- for scale $\varepsilon: x_{1}=0, y_{1}=m\left(f_{1}-a\right)$;

- for scale $Q: x_{2}=L, y_{2}=n\left(f_{2}-b\right)$;

- for scale $P$ : $x_{3}=\frac{m \cdot L}{m+n}, y_{3}=\frac{m \cdot n}{m+n}\left(f_{3}-a-b\right)$.

We assume that the parameters $a$ and $b$ are equal to zero. Further we determine the value of the parameter $m$ from the condition that the height of the entire nomogram should be $100 \mathrm{~mm}$ (according to the assumptions made above). Consequently,

$$
100=0.5325 m\left(\varepsilon_{\max }-\varepsilon_{\min }\right)=0.5325 m \cdot 20 \text {. Hence } m=9.4 .
$$

In a similar way we define the parameter $n$ :

$$
100=-0.3175 n\left(Q_{\max }-Q_{\min }\right)=-0.3175 n \cdot 100 \text {. Hence } n=-3.1 \text {. }
$$

Then the corresponding equation of the scales will take the following form:

$$
x_{1}=0, y_{1}=5.006 \varepsilon ; x_{2}=80, y_{2}=0.9843 Q ; x_{3}=119.4, y_{3}=4.625 P \text {. }
$$

Therefore, the distance between scale $\varepsilon$ and $P$ is $119.4 \mathrm{~mm}$, between $\varepsilon$ and $Q$ it is $80 \mathrm{~mm}$. According to the standard technique [9], based on he obtained experimental data, a nomogram is constructed that has the form (Fig. 3).

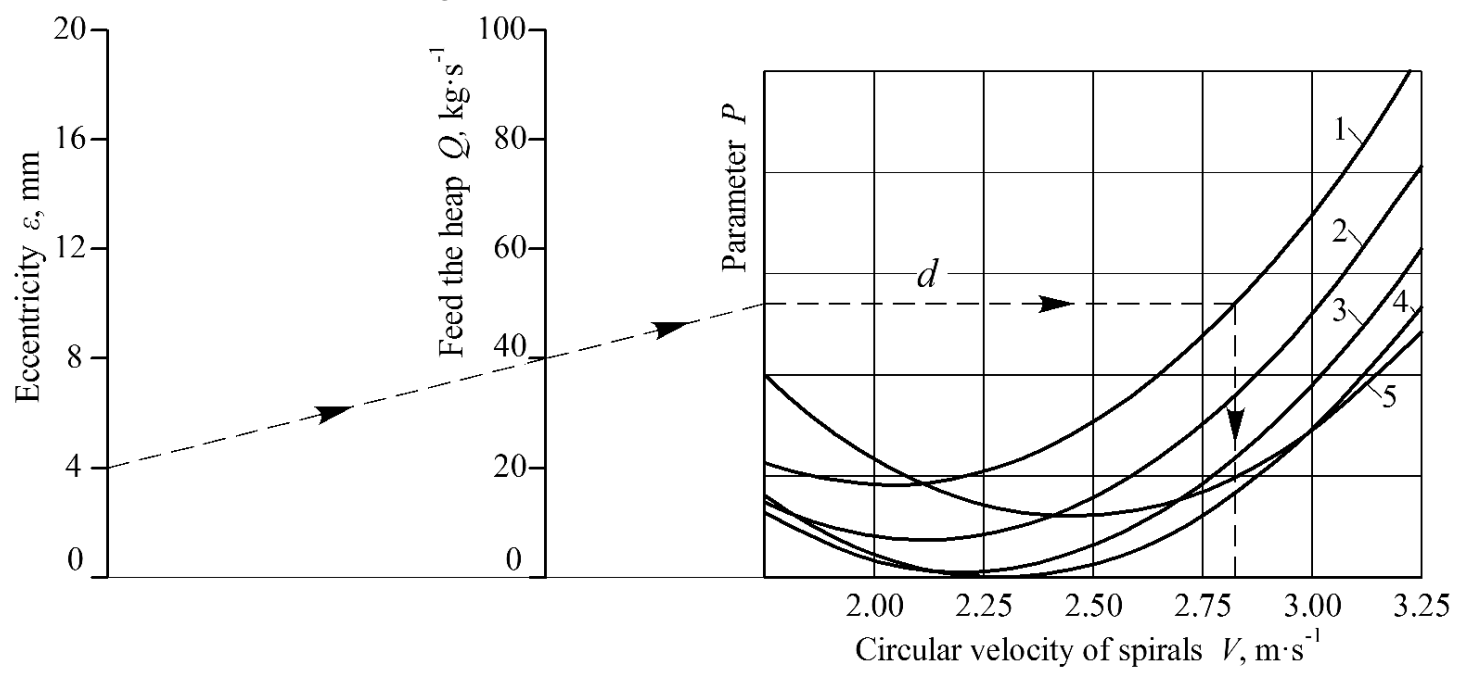

Fig. 3. Nomogram for determining the structural and kinematic parameters of a potato cleaner from admixtures of a spiral type with the angle of inclination $\alpha$ of its spirals to the horizon:

$$
1-\alpha=0^{\circ} ; 2-\alpha=5^{\circ} ; 3-\alpha=10^{\circ} ; 4-\alpha=15^{\circ} ; 5-\alpha=20^{\circ}
$$

In practical use of the obtained nomogram, to determine the parameters of the spiral-type potato cleaner, it is necessary, when setting the eccentricity value $\varepsilon$ and the supply of the material $Q$, to draw a straight line, moving through these two points until it intersects with the axis of the parameter $P$. Next, projecting the value from the axis $P$ (beam $d$ ) onto the preferred curve (1-5) of the angle of inclination $\alpha$ of the cleaner to the horizon, we can determine the required peripheral speed $V$ of its spirals that is necessary to ensure high performance. In the case when beam $d$ intersects several curves 
(1-5) of the angle of inclination $\alpha$ of the cleaner to the horizon, we have a possibility to choose the most technically feasible option.

If we initially set the peripheral speed $V$ of the spirals, we can build in the reverse order the previously described projection connections and arrive at the supply parameters of the potatoes and their admixtures $Q$ and the necessary eccentricity $\varepsilon$ of fastening the spirals of the potato cleaner.

\section{Conclusions}

1. Based on the data of the conducted experimental field research, a model of a full-factor experiment has been constructed, the statistical processing of the results of which made it possible to perform the correlation and regression analyses of the obtained data, applying the Microsoft Excel application program.

2. The conducted research made it possible to build a nomogram that allows determining the optimal design and kinematic parameters of a potato cleaner from the spiral-type admixtures that can be used in designing and development of new potato harvesters.

\section{References}

[1] Klindtworth M. 2016. Potato Technology. Jahrbuch Agritechnik (Frerichs L. Edit.)., 2015. pp. 1-12.

[2] Shpaar D. 2004. Potatoes. Minsk, 465 p. (In Russian).

[3] Bulgakov V., Ivanovs S., Adamchuk V., Ihnatiev Y. Investigation of the influence of the parameters of the experimental spiral potato heap separator on the quality of work. Agronomy Research, 2017. 15(1), pp. 44-54.

[4] Feller R., Margolin E., Hetzroni A., Galili N. Impingement angle and product interference effects on clod separation. Transactions of the american society of agricultural engineers, 1987, 30(2), pp. 357- 360 .

[5] Feng B., Sun, W., Shi L., Sun B., Zhang T., Wu, J. Determination of restitution coefficient of potato tubers collision in harvest and analysis of its influence factors. Nongye Gongcheng Xuebao/Transactions of the chinese society of agricultural engineering, 2017, 33(13), pp.50 -57.

[6] Zaltzman A., Schmilovitch Z. Evolution Of The Potato Fluidized Bed Medium Separator. Summer Meeting American Society Of Agricultural Engineers, Engineering the future harnessing nature through technology, 1985. $27 \mathrm{p}$.

[7] Wei H., Wang D., Lian W., Shao S., Yang X., Huang X. Development Of 4ufg1400 Type Potato Combine Harvester. Transactions O The Chinese Society Of Agricultural Engineering 2013, Vol. 29(1), pp. 11- 17.

[8] Петров Г. Машины для уборки картофеля (Potato Harvesting Machines). Moskow: Mashinostroenije, 2004. 320 p. (In Russian).

[9] Thornley J.H.M., France, J. 2007. Mathematical models in agriculture: Quantitative methods for the plant, animal and ecological sciences. Cabi, $906 \mathrm{pp}$.

[10] Ivanovs S., Adamovics A., Rucins A., Bulgakov V. Investigations into losses of biological mass and quality during harvest of industrial hemp. Engineering for Rural Development, Vol.13, 2014, Jelgava, Latvia, pp. 19-23.

[11]Bulgakov V., Pascuzzi S., Nikolaenko S., Santoro F., Anifantis A. Sotirios, Olt J. Theoretical study on sieving of potato heap elements in spiral separator. Agronomy Research, 2019, Volume 17, No 1. pp. 33-38.

[12]Ногин В.Д., Протодьяконов Н.О., Евлампиев И.И. основы теории оптимизации (The basics of optimization theory). Москва: Высшая школа, 1986. 146 с. (In Russian)

[13] Binghman N., John M. Linear models in statistics. 2010. 255 p.

[14]Мельников С.В. Планирование эксперимента в исследованиях сельскохозяйственных процессов (Planning an experiment in agricultural research). Ленинград: Колос, 1980. 168 p. (In Russian). 